\title{
Relation between structures of naphthalenylchalcone derivatives and their cytotoxic effects on HCT116 human colon cancer cells
}

\author{
Jihyun Park ${ }^{1}$ Soon Young Shin ${ }^{2} \cdot$ Dongsoo $\mathrm{Koh}^{3}$. \\ Young Han Lee $^{2} \cdot$ Yoongho Lim ${ }^{1}$
}

Received: 18 January 2018/Accepted: 23 February 2018/Published online: 6 March 2018

(C) The Korean Society for Applied Biological Chemistry 2018

\begin{abstract}
To find potent chemotherapeutic agents, cytotoxic effects of 42 synthetic chalcone derivatives bearing naphthyl groups on HCT116 human colon cancer cell lines were tested using the clonogenic long-term survival assay. The relationships between their half-maximal cell growth inhibitory concentrations $\left(\mathrm{GI}_{50}\right)$ and structural properties were obtained using comparative molecular field analysis and comparative molecular similarity indices analysis. The structural conditions that showed maximum cytotoxic effects on the colon cancer cells were determined. In addition, a derivative, (E)-1-(2-hydroxy-4,5-dimethoxyphenyl)-3-(naphthalen-1-yl)prop-2-en-1-one, showing the best $\mathrm{GI}_{50}$ value, was assessed for stimulating reactive oxygen species (ROS) production. While its treatment on non-tumorigenic epithelial MCF-12A cell line did not affect the intracellular ROS levels, its treatment on MDAMB-231 human breast cancer cell line showed ROS accumulation. These findings demonstrate that naphthalenylchalcones can be developed as potent chemotherapeutic agents.
\end{abstract}

Electronic supplementary material The online version of this article (https://doi.org/10.1007/s13765-018-0356-6) contains supplementary material, which is available to authorized users.

Yoongho Lim

yoongho@konkuk.ac.kr; yoongholim@gmail.com

1 Division of Bioscience and Biotechnology, BMIC, Konkuk University, Seoul 05029, Republic of Korea

2 Department of Biological Science, Konkuk University, Seoul 05029, Republic of Korea

3 Department of Applied Chemistry, Dongduk Women's University, Seoul 136-714, Republic of Korea
Keywords Clonogenicity · Naphthalenylchalcone . Reactive oxygen species generation - Quantitatively structure-activity relationships

\section{Introduction}

Flavonoids share common features with chalcone, 1,3diphenylprop-2-en-1-one, such as a C6-C3-C6 skeleton (Supplementary Materials Fig. 1A) [1, 2]. However, unlike flavonoids, which consist of a closed ring of $\mathrm{C} 3$, chalcone bears an $\alpha, \beta$-unsaturated carbonyl group. Because various substituents can be attached to both phenyl rings, many chalcone derivatives exist, and they show diverse biological activities: anticancer, antimicrobial, antifungal, antiinflammatory, anti-tuberculosis, and anti-malarial effects [3-8]. Even though there are many chemotherapeutic agents, more potent and safe agents are being developed. An additional benzene ring in chalcone increases its cell permeability. Accordingly, we designed and synthesized the following chalcone derivatives: 3-naphthyl-1-phenylprop-2-en-1-one (Supplementary Materials Fig. 1B) and 1-naphthyl-3-phenyl-prop-2-en-1-one (Supplementary Materials Fig. 1C). There are many methods to measure cytotoxic effects of drugs on cancer cell lines [9-11]. Among them, the clonogenic long-term survival assay requires long experimental times, but it can distinguish between cytotoxic effects of compounds with similar structures [12]. Therefore, we tested the synthesized naphthalenylchalcone derivatives with this assay. Prop-2en-1-one moiety contained in chalcone can act as a Michael acceptor [13]. Our previous experiments demonstrated that a compound with Michael acceptor causes reactive oxygen species (ROS) generation [14]. Therefore, 
we evaluated whether (E)-1-(2-hydroxy-4,5-dimethoxyphenyl)-3-(naphthalen-1-yl)prop-2-en-1-one, showing maximum cytotoxicity among the derivatives tested, causes ROS generation. In addition, the relationships between the physicochemical properties of naphthalenylchalcone derivatives and their cytotoxic effects on HCT116 human colon cancer cells were elucidated using comparative molecular field analysis and comparative molecular similarity indices analysis. The findings obtained from these relationships will give us information regarding the optimum structural conditions to develop potent chemotherapeutic agents containing naphthalenylchalcone moiety.

\section{Materials and methods}

The synthetic procedures to obtain naphthalenylchalcone derivatives have been described in the methods reported previously $[15,16]$. Their cytotoxic effects on HCT116 human colon cancer cells were measured using a clonogenic long-term survival assay [12]. Detailed procedure was followed from the methods reported previously [17]. The clonogenic assay data of 42 naphthalenylchalcone derivatives were obtained at five different concentrations $(0,5,10,20$, and $40 \mu \mathrm{M}$; Fig. 1). The clonogenic densities were measured using a densitometer (MultiGauge, Fujifilm, Japan), and their half-maximal cell growth inhibitory concentrations $\left(\mathrm{GI}_{50}\right)$ were calculated using SigmaPlot (SYSTAT, Chicago, IL) [18]. The relationships between the physicochemical properties of naphthalenylchalcone derivatives and their cytotoxic effects were obtained from comparative molecular field analysis (CoMFA) and comparative molecular similarity indices analysis (CoMSIA), using the Sybyl 7.3 program (Tripos, St. Louis, MO) [19]. Intracellular ROS levels were detected after incubation with $10 \mu \mathrm{M} 2^{\prime}, 7^{\prime}$-dichlorofluorescin diacetate (DCF-DA; Sigma-Aldrich), using a FACSCalibur flow cytometer (Becton-Dickinson Immunocytometry Systems, San Jose, CA), as described previously [14]. Statistical analyses were carried out using a one-way analysis of variance (ANOVA), followed by Kruskal-Wallis test using GraphPad InStat software (La Jolla, CA). All experiments were performed in triplicates [20]. The structures and names of 42 naphthalenylchalcone derivatives are listed in Supplementary Materials Table 1. While derivatives 1-30 contain 3-naphthyl-1-phenyl-prop-2-en-1-one, derivatives 31-42 contain 1-naphthyl-3-phenyl-prop-2-en-1-one. Their structures are summarized in Supplementary Materials Fig. 2. The three-dimensional (3D) structures of derivatives 1-30 were based on the crystallographic structure of derivative 20 [21], and the 3D of derivatives 31-42 were based on the crystallographic structure of derivative $\mathbf{3 4}$ [22]. They were subjected to energy minimization using the Sybyl program, as reported previously $[18,19]$.

\section{Results and discussion}

The $\mathrm{GI}_{50}$ values of 42 naphthalenylchalcone derivatives ranged from 1.02 (derivative 4, $(E)$-1-(2-hydroxy-4,5-dimethoxyphenyl)-3-(naphthalen-1-yl)prop-2-en-1-one) to 96.6 $\mu \mathrm{M}$ (derivative 17, (E)-1-(2-hydroxy-4,6-dimethoxyphenyl)3-(4-methoxynaphthalen-1-yl)prop-2-en-1-one). The negative logarithmic scale of $\mathrm{GI}_{50}$ values $\left(\mathrm{pGI}_{50}\right.$ ) was used as the biological data for the quantitative structure-activity relationships (QSAR) calculation (Supplementary Materials Table 1). Forty-two derivatives were separated into a training set to build the QSAR model, and a test set to validate the QSAR model. Nine derivatives $(\mathbf{8}, \mathbf{1 2}, \mathbf{1 5}, \mathbf{1 9}, \mathbf{2 1}, \mathbf{2 7}, \mathbf{2 9}$, 30, and 40) were chosen for the test set arbitrarily and were validated using hierarchical clustering analysis. As shown in Supplementary Materials Fig. 3, they belong to separate structural groups. Thirty-three derivatives of the training set were aligned to identify interactions between probe atoms and remainder of the derivatives, using the Sybyl/DATABASE Alignment module (Supplementary Materials Fig. 4). The QSAR was analyzed using a CoMFA model, which provides information about steric and electrostatic field descriptors, as described in a previous report [23]. Of many CoMFA models generated using partial least-squares regression and region focusing method, the model showing the best cross-validation correlation coefficient $\left(q^{2}\right)$ of 0.529 was chosen. According to this model, the non-cross-validated coefficient $\left(r^{2}\right)$, the optimal number of components, the standard error of estimate, and the $F$ value were determined to be $0.978,6,0.068$, and 194.814 , respectively. The $\mathrm{pGI}_{50}$ values, predicted using this model, were compared to the experimental data. As listed in Supplementary Materials Table 2, the residuals between the two values ranged from 0.34 to $7.55 \%$. Because the test set was prepared to validate the CoMFA model, the $\mathrm{pGI}_{50}$ values of the test set were calculated using this model, and the predicted values were compared to the experimental data. Their residuals ranged between 2.65 and $23.32 \%$. As a result, this CoMFA model can be used to explore relationships between the physicochemical properties of 42 naphthalenylchalcone derivatives and their cytotoxic effects on HCT116 human colon cancer cells. The plot of experimental data versus predicted values is shown in Supplementary Materials Fig. 5.

The same procedures were performed to generate CoMSIA models, which provide information about the steric and electrostatic field descriptors, as well as the hydrophobic, hydrogen bond (H-bond) donor and acceptor fields. Out of many models, the CoMSIA model, which shows the best $\mathrm{q}^{2}$ of 0.504 , was selected, where $\mathrm{r}^{2}$, the 
Fig. 1 Clonogenic long-term survival assays of 42

naphthalenylchalcone

derivatives at five different

concentrations: $0,5,10,20$ and

$40 \mu \mathrm{M}$
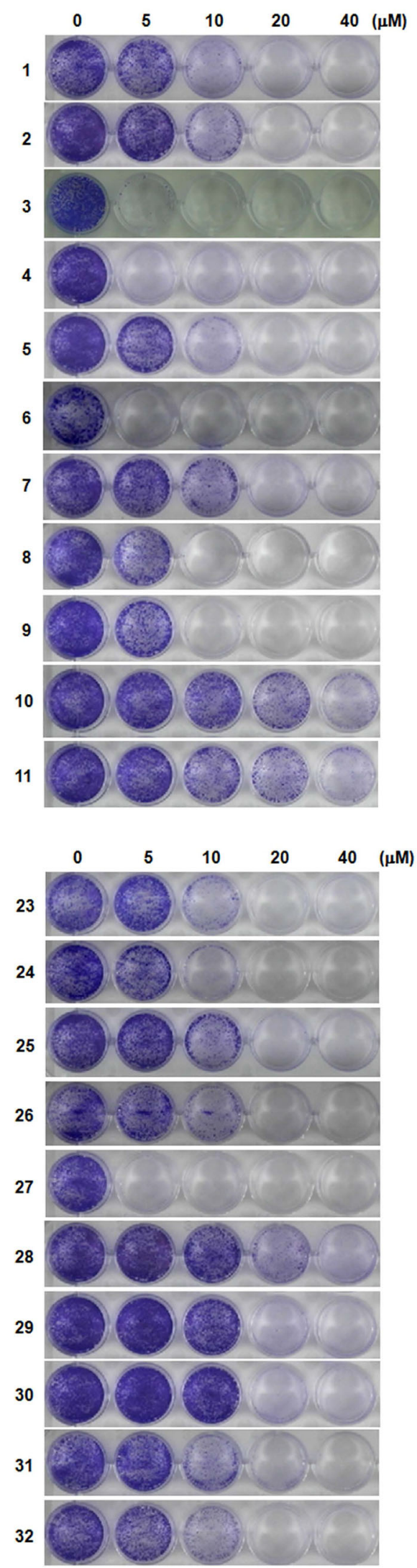
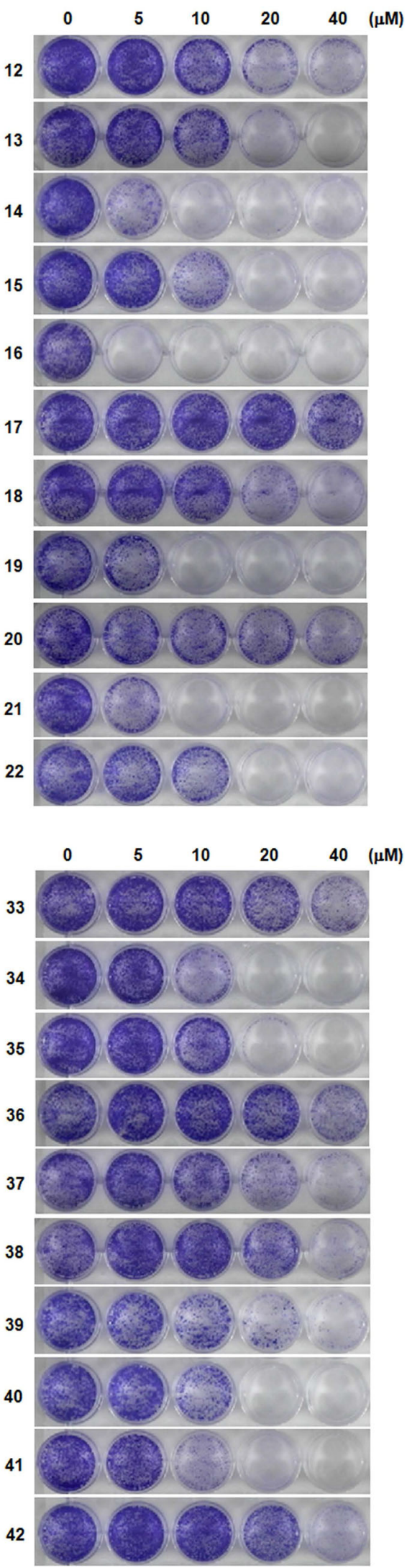
optimal number of components, the standard error of estimate, and $F$ value were determined to be $0.937,6$, 0.116, and 64.272, respectively. This CoMSIA model includes steric and hydrophobic fields. The residuals between experimental values of $\mathrm{pGI}_{50}$ of the training set and values predicted using this model ranged from 0.12 to $12.74 \%$. Likewise, the residuals for the test set ranged between 6.35 and $25.08 \%$. They are listed in Supplementary Materials Table 2. Therefore, this CoMSIA model is reliable. The plot of experimental data versus predicted values is shown in Supplementary Materials Fig. 6.

To visualize the results obtained from the CoMFA model, its contour maps were generated using the Sybyl program, where steric and electrostatic field descriptors contributed to 51.3 and $48.7 \%$, respectively. The steric field descriptors could be divided into a bulky favored region (80\%) and a bulky disfavored region (20\%), as shown in Supplementary Materials Fig. 7, where derivative
4 was inserted as a template. While the existence of an additional benzene ring at the 3-phenyl ring increases the cytotoxic effects, its existence at 1-phenyl ring decreases the cytotoxic effect. The activities of derivatives 1-30 with 3-naphthyl-1-phenyl-prop-2-en-1-one are better than those of derivatives 31-42 with 1-naphthyl-3-phenyl-prop-2-en1-one. Because substituents of all naphthalenylchalcone derivatives, except derivative $\mathbf{4 2}$, vary in the number or position of methoxy groups, the electrostatic field descriptors generated by the CoMFA model do not discriminate between the pharmacophores. Like the CoMFA model, the contour maps by the CoMSIA model were generated using Sybyl. As mentioned above, the CoMSIA model includes the steric and hydrophobic fields. The contour maps of the steric field descriptors are same as those from the CoMFA model. The hydrophobic field descriptors could be divided into a favored region (88\%) and a disfavored region (12\%), as shown in Supplementary
Fig. 2 MDA-MB-231 human breast cancer cells and nontumorigenic epithelial MCF$12 \mathrm{~A}$ cell lines were incubated with $10 \mathrm{mmol} / \mathrm{L}$ DCF-DA for $60 \mathrm{~min}$, followed by the addition of $10 \mu \mathrm{M}$ derivative 4 or $10 \mu \mathrm{M}$ DPP23 for $1 \mathrm{~h}$. Fluorescence was then measured using a FACSCalibur
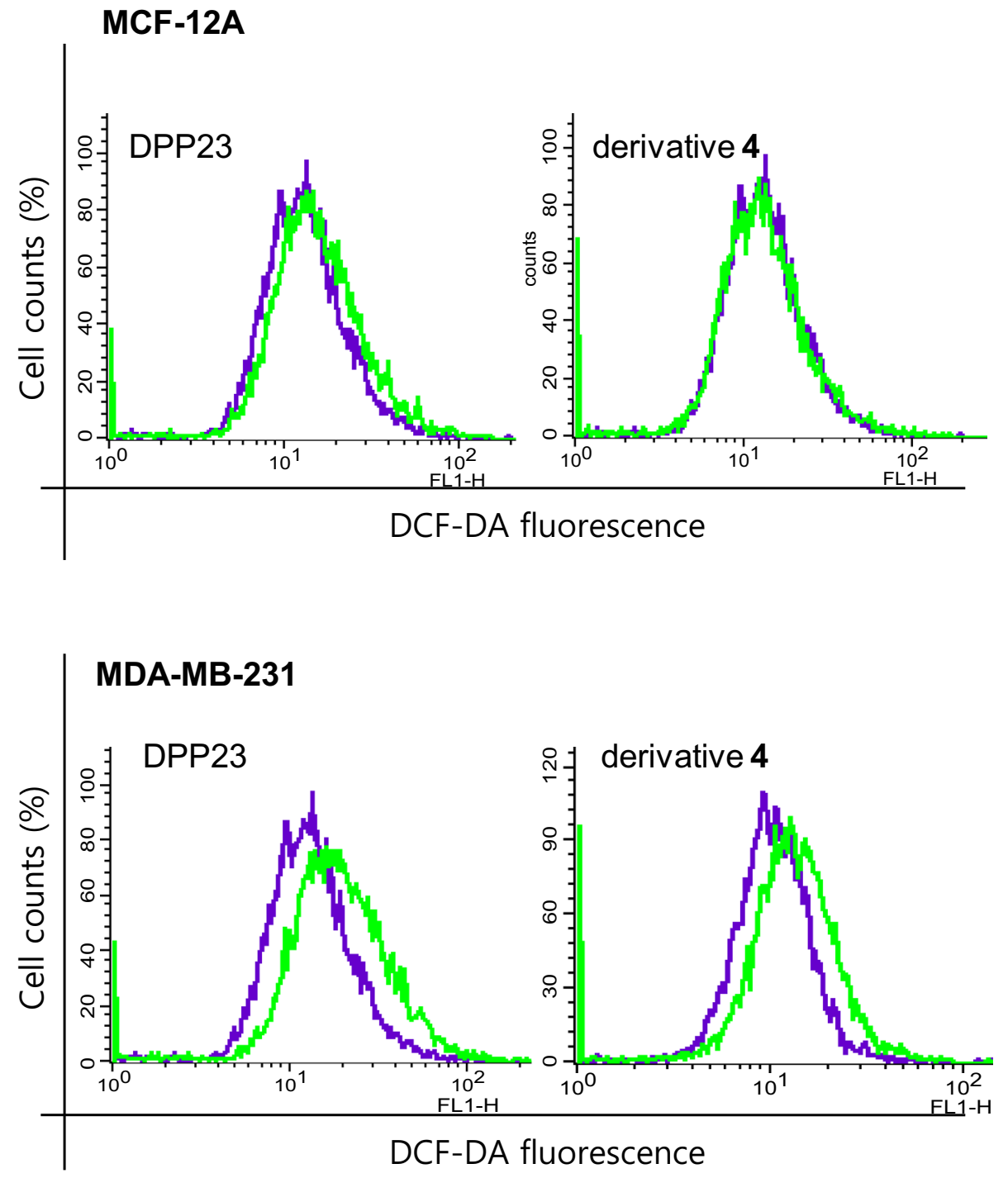
Materials Fig. 8, where derivative $\mathbf{4}$ was inserted as a template. The hydrophobic groups at $\mathrm{R}_{2}, \mathrm{R}_{5}$, and $\mathrm{R}_{8}$, shown in Supplementary Materials Fig. 2, decrease the activities. Derivatives 8, 14, and 26 with methoxy groups at $\mathrm{R}_{2}$ position show better activities than derivatives $7, \mathbf{1 3}$, and 25, respectively. Derivatives 19, 21, 22, 23, 24, and 40 with methoxy groups at $\mathrm{R}_{5}$ position show better activities than derivatives $7,9,10,11,12$, and 31, respectively. Derivative 37 with methoxy group at $\mathrm{R}_{8}$ position shows better activity than derivative 38. Derivative $\mathbf{4 2}$ with hydroxy group at $\mathbf{R}_{8}$ position shows better activity than derivative $\mathbf{3 6}$.

All derivatives include the Michael acceptor, as marked in Supplementary Materials Fig. 2. Because the compounds containing the Michael acceptor could generate ROS, derivative 4 showing the best $\mathrm{GI}_{50}$ value was assessed for stimulating ROS production. A chalcone, $(E)-3-$ (3,5-dimethoxyphenyl)-1-(2-methoxyphenyl)prop-2-en-1one (named as DPP23), was proved to generate ROS in our previous experiments [14]. Therefore, DPP23 was used as a reference compound. The intracellular ROS levels were measured using DCF-DA fluorescence, which indicates ROS accumulation. The treatment of DPP23 on non-tumorigenic epithelial MCF-12A cell line did not affect the intracellular ROS levels, as shown in Fig. 2 (left top). However, its treatment on MDA-MB-231 human breast cancer cell line showed ROS accumulation, as shown in Fig. 2 (left bottom). Similarly, derivative 4 was added to both non-tumorigenic epithelial MCF-12A cell line and MDA-MB-231 human breast cancer cell line. Like DPP23, while the treatment of derivative 4 on non-tumorigenic epithelial MCF-12A cell line did not affect the intracellular ROS levels, as shown in Fig. 2 (right top), its treatment on MDA-MB-231 human breast cancer cell line showed ROS accumulation, as shown in Fig. 2 (right down). As a result, we confirm that derivative $4,(E)$-1-(2-hydroxy-4,5-dimethoxyphenyl)-3-(naphthalen-1-yl)prop-2-en-1-one, is a ROS generating naphthalenylchalcone.

$2^{\prime}$-Hydroxy-2,3,5'-trimethoxychalcone induces apoptosis in breast cancer cell lines [24]. 2-Hydroxy-3 $3^{\prime}, 5,5^{\prime}$ trimethoxychalcone inhibits nuclear factor-kappaB (NF$\kappa \mathrm{B})$-mediated $\mathrm{GRO} \alpha$ expression and prevents invasion of MDA-MB-231 human breast cancer cells [25]. 2'-Hydroxy-2,4,6-trimethoxy-5', $6^{\prime}$-naphthochalcone disturbs the microtubular network of colon cancer cells, which results in inducing $\mathrm{G} 2 / \mathrm{M}$ cell cycle arrest and apoptosis [26]. 2-Hydroxy-4-methoxy-2', $3^{\prime}$-benzochalcone inhibits tubulin polymerization [27]. They all belong to the group of synthetic chalcones. As listed above, various chalcones with methoxy or naphthyl groups have been known to show anticancer effects [28-31]. However, anticancer effects of chalcones with naphthyl group have rarely been studied, and the relationships between their structural properties and cytotoxic effects have not been reported.
In this research, the clonogenic long-term survival effects of 42 naphthalenylchalcone derivatives on HCT116 human colon cancer cells were measured. The relationships between their cytotoxic effects and physicochemical properties, obtained using comparative molecular field analysis and comparative molecular similarity indices analysis, demonstrated the optimal structural conditions that show maximum cytotoxic effects on colon cancer cells. In addition, ROS generation was measured for the title compound of the current research, $(E)-1-(2$-hydroxy4,5-dimethoxyphenyl)-3-(naphthalen-1-yl)prop-2-en-1one, which showed the maximum half-maximal cell growth inhibitory effect. As expected from previous studies, the compound showed ROS generation. Thus, these findings demonstrated that naphthalenylchalcones can be developed as potent chemotherapeutic agents.

Acknowledgments This work was supported by the Priority Research Centers Program (NRF, 2009-0093824) and written as part of Konkuk University's research support program for its faculty on sabbatical leave in 2016.

\section{References}

1. Havsteen BH (2002) The biochemistry and medical significance of the flavonoids. Pharmacol Ther 96:67-202

2. Jung H, Ahn S, Park M, Yoon H, Noh HJ, Kim SY, Yoo JS, Koh D, Lim Y (2015) 1H and 13C NMR spectral assignments of 18 novel polymethoxylated naphthochalcones bearing pyrazoline-1carbothioamide groups. Magn Reson Chem 53:383-390

3. Mahapatra DK, Bharti SK, Asati V (2015) Anti-cancer chalcones: structural and molecular target perspectives. Eur J Med Chem 98:69-114

4. Feng L, Maddox MM, Alam MZ, Tsutsumi LS, Narula G, Bruhn DF, Wu X, Sandhaus S, Lee RB, Simmons CJ, Tse-Dinh YC, Hurdle JG, Lee RE, Sun D (2014) Synthesis, structure-activity relationship studies, and antibacterial evaluation of 4-chromanones and chalcones, as well as olympicin A and derivatives. J Med Chem 57:8398-8420

5. de Sa NP, Cisalpino PS, Tavares Lde C, Espindola L, Pizzolatti MG, Santos PC, de Paula TP, Rosa CA, de Souza Dda G, Santos DA, Johann S (2015) Antifungal activity of 6-quinolinyl N-oxide chalcones against Paracoccidioides. J Antimicrob Chemother 70:841-845

6. Wu J, Li J, Cai Y, Pan Y, Ye F, Zhang Y, Zhao Y, Yang S, Li X, Liang G (2011) Evaluation and discovery of novel synthetic chalcone derivatives as anti-inflammatory agents. J Med Chem 54:8110-8123

7. Chiaradia LD, Martins PG, Cordeiro MN, Guido RV, Ecco G, Andricopulo AD, Yunes RA, Vernal J, Nunes RJ, Terenzi H (2012) Synthesis, biological evaluation, and molecular modeling of chalcone derivatives as potent inhibitors of Mycobacterium tuberculosis protein tyrosine phosphatases (PtpA and PtpB). J Med Chem 55:390-402

8. Sharma N, Mohanakrishnan D, Shard A, Sharma A, Saima Sinha AK, Sahal D (2012) Stilbene-chalcone hybrids: design, synthesis, and evaluation as a new class of antimalarial scaffolds that trigger cell death through stage specific apoptosis. J Med Chem $55: 297-311$ 
9. Riss TL, Moravec RA (2004) Use of multiple assay endpoints to investigate the effects of incubation time, dose of toxin, and plating density in cell-based cytotoxicity assays. Assay Drug Dev Technol 2:51-62

10. Fan F, Wood KV (2007) Bioluminescent assays for highthroughput screening. Assay Drug Dev Technol 5:127-136

11. Decker T, Lohmann-Matthes ML (1988) A quick and simple method for the quantitation of lactate dehydrogenase release in measurements of cellular cytotoxicity and tumor necrosis factor (TNF) activity. J Immunol Methods 115:61-69

12. Franken NA, Rodermond HM, Stap J, Haveman J, van Bree C (2006) Clonogenic assay of cells in vitro. Nat Protoc 1:2315-2319

13. Dinkova-Kostova AT, Abeygunawardana C, Talalay P (1998) Chemoprotective properties of phenylpropenoids, bis(benzylidene)cycloalkanones, and related Michael reaction acceptors: correlation of potencies as phase 2 enzyme inducers and radical scavengers. J Med Chem 41:5287-5296

14. Shin SY, Lee JM, Lee MS, Koh D, Jung H, Lim Y, Lee YH (2014) Targeting cancer cells via the reactive oxygen speciesmediated unfolded protein response with a novel synthetic polyphenol conjugate. Clin Cancer Res 20:4302-4313

15. Koh D, Jung Y, Kim BS, Ahn S, Lim Y (2016) H and C NMR spectral assignments of naphthalenyl chalcone derivatives. Magn Reson Chem 54:842-851

16. Hwang D, Hyun J, Jo G, Koh D, Lim Y (2011) Synthesis and complete assignment of NMR data of 20 chalcones. Magn Reson Chem 49:41-45

17. Shin SY, Yoon H, Hwang D, Ahn S, Kim DW, Koh D, Lee YH, Lim Y (2013) Benzochalcones bearing pyrazoline moieties show anti-colorectal cancer activities and selective inhibitory effects on aurora kinases. Bioorg Med Chem 21:7018-7024

18. Yoon H, Kim TW, Shin SY, Park MJ, Yong Y, Kim DW, Islam T, Lee YH, Jung KY, Lim Y (2013) Design, synthesis and inhibitory activities of naringenin derivatives on human colon cancer cells. Bioorg Med Chem Lett 23:232-238

19. Jung Y, Shin SY, Yong Y, Jung H, Ahn S, Lee YH, Lim Y (2015) Plant-derived flavones as inhibitors of aurora B kinase and their quantitative structure-activity relationships. Chem Biol Drug Des 85:574-585

20. Jung H, Shin SY, Jung Y, Tran TA, Lee HO, Jung KY, Koh D, Cho SK, Lim Y (2015) Quantitative relationships between the cytotoxicity of flavonoids on the human breast cancer stem-like cells MCF7-SC and their structural properties. Chem Biol Drug Des 86:496-508
21. Ahn S, Lim Y, Koh D (2015) Crystal structure of 2-(2,3-dimethoxy-naphthalen-1-yl)-3-hydroxy-6-methoxy-4H-chromen-4one. Acta Crystallogr E Crystallogr Commun 71:0842-0843

22. Koh D (2014) Crystal structure of (E)-3-(2,4-dimethoxyphenyl)1-(1-hydroxynaphthalen-2-yl)prop-2-en-1-one. Acta Crystallogr Sect E: Struct Rep Online 70:o1034-01035

23. Hyun J, Shin SY, So KM, Lee YH, Lim Y (2012) Isoflavones inhibit the clonogenicity of human colon cancer cells. Bioorg Med Chem Lett 22:2664-2669

24. da Lee H, Jung Jung Y, Koh D, Lim Y, Lee YH, Shin SY (2016) A synthetic chalcone, 2'-hydroxy-2,3,5'-trimethoxychalcone triggers unfolded protein response-mediated apoptosis in breast cancer cells. Cancer Lett 372:1-9

25. da Lee Y, da Lee H, Jung JY, Koh D, Kim GS, Ahn YS, Lee YH, Lim Y, Shin SY (2016) A synthetic chalcone derivative, 2-hydroxy-3',5,5'-trimethoxychalcone (DK-139), suppresses the TNF $\alpha$-induced invasive capability of MDA-MB-231 human breast cancer cells by inhibiting NF- $\kappa B$-mediated GROalpha expression. Bioorg Med Chem Lett 26:203-208

26. Lee JM, Lee MS, Koh D, Lee YH, Lim Y, Shin SY (2014) A new synthetic $\quad 2^{\prime}$-hydroxy-2,4,6-trimethoxy-5', $6^{\prime}$-naphthochalcone induces $\mathrm{G} 2 / \mathrm{M}$ cell cycle arrest and apoptosis by disrupting the microtubular network of human colon cancer cells. Cancer Lett 354:348-354

27. Shin SY, Kim JH, Yoon H, Choi YK, Koh D, Lim Y, Lee YH (2013) Novel antimitotic activity of 2-hydroxy-4-methoxy-2', $3^{\prime}$ benzochalcone (HymnPro) through the inhibition of tubulin polymerization. J Agric Food Chem 61:12588-12597

28. Rao CM, Yejella RP, Rehman RS, Basha SH (2015) Molecular docking based screening of novel designed chalcone series of compounds for their anti-cancer activity targeting EGFR kinase domain. Bioinformation 11:322-329

29. Sharma R, Dhingra N, Patil S (2016) CoMFA, CoMSIA, HQSAR and molecular docking analysis of ionone-based chalcone derivatives as antiprostate cancer activity. Indian J Pharm Sci 78:54-64

30. Ivkovic BM, Nikolic K, Ilic BB, Zizak ZS, Novakovic RB, Cudina OA, Vladimirov SM (2013) Phenylpropiophenone derivatives as potential anticancer agents: synthesis, biological evaluation and quantitative structure-activity relationship study. Eur J Med Chem 63:239-255

31. Kumar V, Kumar S, Hassan M, Wu H, Thimmulappa RK, Kumar A, Sharma SK, Parmar VS, Biswal S, Malhotra SV (2011) Novel chalcone derivatives as potent Nrf2 activators in mice and human lung epithelial cells. J Med Chem 54:4147-4159 